\title{
ASPECTOS SEDIMENTOLÓGICOS E BATIMÉTRICOS DA PLATAFORMA CONTINENTAL NORTE DE PERNAMBUCO - BRASIL
}

\author{
Maria Cristina da Silva Sales de Melo; \\ Roberto Lima Barcellos ${ }^{1}$; \\ Liza Ellen Eurico de Oliveira ${ }^{2}$; \\ Valdir do Amaral Vaz Manso ${ }^{2}$
}

10.18190/1980-8208/estudosgeologicos.v29n2p17-30

${ }^{1}$ Laboratório de Oceanografia Geológica - DOCEAN/UFPE;

cristinasales.mms@gmail.com; roberto.barcellos@ufpe.br

${ }^{2}$ Laboratório de Geologia de Geofísica Marinha - CTG/UFPE;

lizaellendeoliveira@gmail.com; valdiramaralvazmanso

\section{RESUMO}

O presente trabalho apresenta uma contribuição acerca da fisiografia e da cobertura sedimentar da região de plataforma interna e média adjacente à Ilha de Itamaracá (PE). Esta pesquisa se baseou nos dados obtidos do projeto "Granulados Marinhos GRANMAR Pernambuco". A partir desta cobertura de dados foram elaborados mapas de batimetria, do tamanho médio das partículas sedimentares, dos aspectos de seleção do sedimento, e dos teores de carbonato de cálcio dos depósitos superficiais. A análise da fisiografia revelou a presença de reentrâncias e depressões associadas às desembocaduras dos rios. Os principais resultados demonstraram que a cobertura sedimentar é predominantemente calcária de origem bioclástica, com granulação arenosa e que a distribuição dos depósitos superficiais é controlada pela associação de aspectos fisiográficos e hidrodinâmicos locais. Os arenitos de praia e canais atuam na energia do meio, o que promove o a seleção dos sedimentos na plataforma. Enquanto que os paleovales e depressões do fundo marinho atuam como elementos de retenção dos sedimentos mais finos oriundos do continente.

Palavras chave: Ilha de Itamaracá, batimetria, plataforma interna, plataforma média, sedimentologia.

\begin{abstract}
This paper provides a brief contribution about the physiography and sedimentary cover of the inner and middle shelf adjacent to Itamaracá Island (PE). This research was based on data obtained from the project "Granulados Marinhos - GRANMAR Pernambuco". From this data coverage, maps of bathymetry, mean diameter of sedimentary particles, sorting aspects, and calcium carbonate contents of surface deposits were prepared. The analysis of the physiography revealed the presence of indentations and depressions associated with the mouths of the rivers. The main results showed that the sedimentary cover is predominantly limestone of bioclastic origin, with sandy granulation and that the distribution of surface deposits is controlled by the association of local physiographic and hydrodynamic aspects. Beach sandstones and channels act on the environment energy, which promotes the sorting of sediments in the shelf.
\end{abstract}

Keywords: Itamaracá Island, bathymetry, inner shelf, middle shelf, sedimentology. 


\section{INTRODUÇÃO}

As plataformas continentais são ambientes dinâmicos, nos quais a cobertura sedimentar e a fisiografia são reflexos de todas as dinâmicas existentes (Ponzi, 2004). Como definição geral, as plataformas continentais representam extensões submarinas da margem continental (Hezen et al., 1996).

No Brasil, as margens continentais são do tipo passiva, apresentando um relevo aplainado, cuja declividade iniciase desde a região praial até a quebra da plataforma, onde ocorre uma variação abrupta da declividade, em torno dos 130 $\mathrm{m}$ de profundidade (Coutinho, 1996; Suguio, 2003). As características fisiográficas são resultantes da combinação entre a herança geológica local com outras condicionantes ambientais, como os espaços de acomodação, o aporte de sedimentos, a circulação oceânica, além das variações do nível do mar ao longo do tempo (Dominguez, 2009).

Durante o último processo regressivo do nível relativo do mar, o Último Máximo Glacial (UMG), que ocorreu há cerca de 20.000 anos AP, as plataformas brasileiras ficaram expostas, parcial ou totalmente. Esse evento proporcionou a ocorrência de processos erosivos, tanto de origem fluvial quanto subaéreo o que ocasionou a formação de vales fluviais na plataforma (Conti \& Furtado, 2009; Blum et al., 2013; Harris et al., 2014). Os sedimentos que provêm do continente por meio dos rios que percorreram estes vales de plataforma, carreando material para zonas mais distantes. A presença dos vales, associado aos influxos de material terrígeno podem ocasionar a criação de outras feições, como os deltas e cristas arenosas ao longo de toda plataforma (Wagle \& Veerayya, 1996; Gardner et al., 2007; Weschenfelder et al., 2008; Salzmann et al., 2013).

No UMG o nível do mar ficou cerca de $120 \mathrm{~m}$ abaixo do nível atual, o que deixou um grande número de plataformas continentais expostas em todo o globo. Essa exposição afetou a plataforma externa da região de estudo, em decorrência da baixa declividade desta, o que indica que a plataforma ficou sob ação dos condicionantes climáticos e dos processos relacionados à ação de sistemas fluviais, possibilitando a ocorrência de vales na região (Koss et al., 1994; Yokoyama et al., 2000; Clark et al., 2009; Yokoyama et al., 2018; Camargo et al., 2015). No caso da plataforma continental de Pernambuco, cuja quebra está relacionada à cota batimétrica média de 65 m (Araújo et al., 2004; Camargo et al., 2015), o talude superior ficou exposto durante este período.

O presente trabalho objetivou ampliar o conhecimento da fisiografia das regiões de plataforma interna e média, adjacentes à Ilha de Itamaracá, costa centro-norte de Pernambuco, com enfoque nas estruturas morfológicas e na composição da cobertura sedimentar do leito marinho.

\section{ÁREA DE ESTUDO}

A área de estudo representa a folha Itamaracá do projeto "Granulados Marinhos em Pernambuco GRANMAR-PE”, e está localizada entre as coordenadas $7^{\circ} 30^{\prime} 32^{\prime \prime} / 7^{\circ} 59^{\prime} 54^{\prime \prime} \mathrm{S}$ e $34^{\circ} 37^{\prime} 55,2^{\prime \prime} / 34^{\circ} 49^{\prime} 48,2^{\prime \prime} \mathrm{W}$, com uma área de aproximadamente $750 \mathrm{~km}^{2}$. Os pontos de coleta foram dispostos desde a divisa entre os estados de Pernambuco e Paraíba (porção norte da área), ao largo da foz do rio Goiana, e o limite entre as 
cidades de Paulista e Olinda, adjacente à foz do Rio Paratibe (porção sul da área)

A malha amostral foi composta por 40 transectos perpendiculares à linha de costa, com espaçamento aproximado de 1 milha náutica $(1852 \mathrm{~m})$ entre os pontos. Para assegurar uma melhor definição das feições batimétricas, foram incluídos no presente estudos 63 dados de profundidade na região adjacente à Ilha de Itamaracá, obtidos pelo Laboratório de Geologia e Geofísica Marinha da UFPE.

Conforme Flores-Montes et al. (1998), a área de estudo apresenta clima classificado como tropical do tipo Am' conforme a classificação de Köppen, com transição para As', apresentando uma faixa térmica média entre $25^{\circ} \mathrm{C}$ e $27^{\circ} \mathrm{C}$ com oscilação de $\pm 3^{\circ} \mathrm{C}$ durante o de um ano. Apresenta uma precipitação média de $1600 \mathrm{~mm}$, com período chuvoso delimitado entre abril e agosto (CPRH, 2003; Oliveira filho, 2015; Barcellos et al., 2016).

A zona costeira foi densamente recoberta pelo bioma de Mata Atlântica, mas grande parte dessa vegetação foi substituída pela monocultura canavieira e pelo crescimento urbano desordenado. No presente, existem apenas vestígios de mata secundária em decorrência dos impactos antrópicos (SUDENE, 1978; Almeida, 2011).

$\mathrm{Na}$ região destaca-se uma extensa zona estuarina o sistema Ilha de Itamaracá, formado pelos canais de Santa Cruz e Itapessoca/Catuama, onde ocorrem sistemas de manguezais com a presença dos gêneros Avicennia (mangue canoé), Conocarpus (mangue de botão), Laguncularia (mangue manso) e Rhizophora (mangue vermelho) (Silva, 2004).

$\mathrm{Na}$ região marinha ocorre uma dominância de diatomáceas, Chlorophycea e Pheophycea na porção junto à costa, e na direção offshore ocorre abundância de algas calcárias do gênero Lithothamnium, cuja distribuição é controlada pelas condições hidrodinâmicas e pela topografia de fundo (Almeida, 2011; Silva, 2004; Silva, 2012; Varela, 2011).

A área de estudo apresenta influência de três bacias hidrográficas e de um sistema estuarino associado a rios costeiros. A norte situa-se a bacia do rio Goiana, na região central ocorre a bacia do Rio Jaguaribe (Ilha de Itamaracá), e a sul localiza-se a bacia do rio Paratibe.

Todas essas bacias hidrográficas costeiras apresentam grande impacto antrópico, agravado pelo crescimento populacional $(\mathrm{CPRH}, 2001$; Almeda, 2011). O sistema Itamaracá (canais de Santa Cruz e de Itapessoca/Catuama) é formado pela confluência dos rios Aracata, Bonança, Botafogo, Carrapicho, Catuama, Cumbre, Igarassu, Itapessoca, Itapirema, Palmeira, Riacho Jardim, Tabatinga e Utinga. Na porção norte e sul, os afluentes mais importantes são o Botafogo e Igarassu, respectivamente. Ambos sofrem uma grande influência antrópica, e os poluentes neles descarregados (Gaspar 2009; Silva, 2012), chegam a plataforma interna juntamente com a carga sedimentar.

Ratisbona (1976) afirmou que durante $o$ período anual a região apresenta 3 direções de vento dominantes: o período chuvoso com direção SE com média de $3,2 \mathrm{~m} / \mathrm{s}$, período seco com direção NE e média de $2,6 \mathrm{~m} / \mathrm{s}$, e em dezembro, durante o ápice térmico, ENE e com média de 2,0 m/s. As marés da região são do tipo mesomaré, conforme a classificação de Hayes (1979), o que resulta em uma variação de 2 a $4 \mathrm{~m}$. As ondas são dominantemente do tipo sea, com ocorrência de swell. As ondas apresentam altura média de $0,4 \mathrm{~m}$ e período médio de 7,5 s (Barros, et al 2007; Varela, 2010). A temperatura da água varia entre $25,7^{\circ}$ a $30,8^{\circ} \mathrm{C}$ nos períodos secos e chuvosos respectivamente. Outro fator que possui variação sazonal da mesma forma é a 
salinidade que oscila entre 28,9 e 37,2 , e para ambos parâmetros os menores valores são observados mais próximos à costa (Manso et al.,1992; Varela, 2010; Domingues et al., 2017). De acordo com Domingues et al. (2017), a região é classificada como de águas tropicais, caracterizada por temperaturas quentes $\left(>26^{\circ} \mathrm{C}\right)$ e salinas $(>35)$, com gradientes superficiais observados durante o verão, e uma coluna d'água com salinidade e temperatura predominantemente uniforme.
Com base em Medeiros \& Kjerfve (1993) a zona costeira do litoral Norte de Pernambuco é caracterizada por uma série de linhas de recifes paralelas e contínuas, que apresentam abundância de formações coralíneas e algas calcárias que se fixam a um substrato arenoso.

A figura 1 mostra a localização da área de estudo, com os pontos de coleta e os pontos de batimetria já existentes que foram incluídos nesta pesquisa.

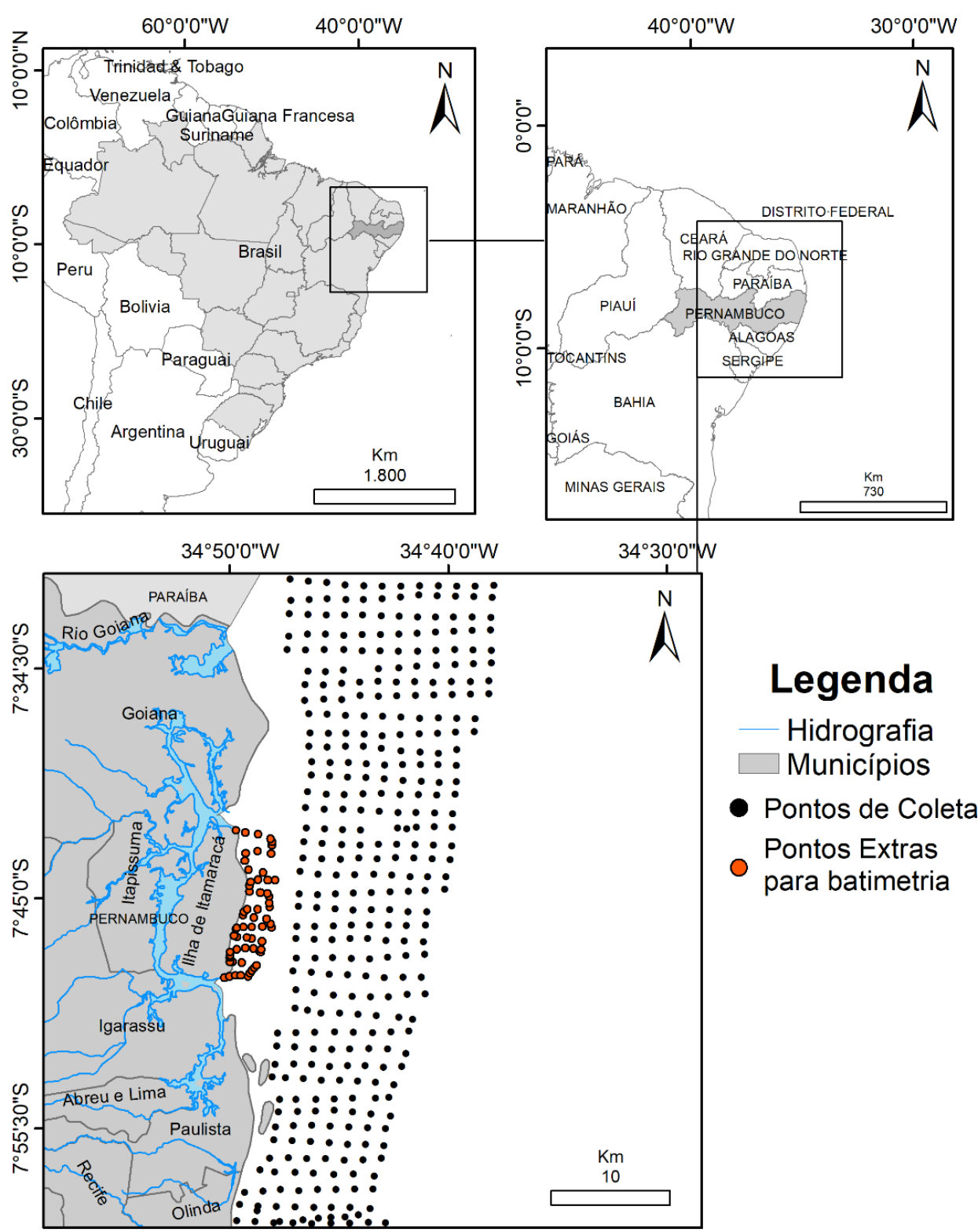

Figura 1 - Localização da área de estudos com os 364 pontos de coleta (em preto), e os 63 pontos de batimetria (em vermelho) coletados por outro projeto e utilizados nesta pesquisa. 


\section{MATERIAL E MÉTODOS}

Os dados tratados nesta pesquisa foram coletados pela equipe do "Projeto GRANMAR Brasil", dentro do qual a área de estudo foi tratada pelo subprojeto: "Avaliação do potencial mineral dos granulados marinhos no litoral do Brasil$P E / C E$ ", realizado pela Companhia de Pesquisa de Recursos Minerais do Serviço Geológico do Brasil (CPRM, 2018). As coletas de sedimentos foram realizadas em cruzeiros oceanográficos que ocorreram em 2004, e cobriram os 40 perfis mostrados na figura 1 (CPRM, 2018). A coleta totalizou 364 amostras sedimentos superficiais de fundo marinho.

As amostras foram coletadas com o uso de uma draga do tipo "Van Veen". O material foi analisado segundo os métodos de análise granulométrica descritos em Suguio (1973), e foi aplicado o tratamento estatístico conforme proposto por Folk \& Ward (1957) e Larssonneur et al. (1982). Os parâmetros observados, conforme o interesse desta pesquisa, foram os dados de diâmetro médio (phi) e grau de seleção das partículas sedimentares que compõem os depósitos coletados. Os dados de teor de carbonato de cálcio $\left(\mathrm{CaCO}_{3}\right)$ foram obtidos por meio da aferição de peso do material seco em balança semi-analítica, executada antes e após o ataque com $\mathrm{HCl}$ a $10 \%$, de acordo com os métodos descritos em Carver (1971).

Os dados batimétricos utilizados, conforme mencionado antes, foram obtidos pelo projeto. Adicionalmente foram incorporados mais 63 pontos de profundidade adjacentes à ilha de Itamaracá, anteriormente coletados pelo LGGM-UFPE para um melhor refinamento da malha amostral gerada. Os shapefiles, utilizados para elaboração dos mapas do presente trabalho, são da CPRM (2004), georrefrenciados no sistema SAD69, cujo datum é o SIRGAS 2000, para latitude 25S.

\section{RESULTADOS E DISCUSSÃO Fisiografia de fundo}

Com o processamento dos dados de profundidade, foi elaborado um mapa batimétrico da região de estudo (Fig. 2).

Varela (2010) e Almeida (2011, 2018) descreveram a ocorrência de arenitos de praia ao longo de toda costa da área em estudo, estruturas que apresenta algumas falhas (aberturas) longitudinais e perpendiculares ao longo de sua extensão.

Segundo os autores citados, essas estruturas são responsáveis por regular a dinâmica local. Por estarem localizados na plataforma interna, estes arenitos atenuam a ação dos impactos das ondas até o ambiente praial. De acordo com Almeida (2018), as regiões de depressões observadas adjacentes à costa são resultantes da combinação da dinâmica das marés, desembocaduras dos cursos hídricos e das falhas observadas nos arenitos.

Estudo desenvolvido por Camargo et al. (2015), em relação à região sul da plataforma continental de Pernambuco, descreve que as estruturas morfológicas atuais como paleovales e terraços de abrasão por ondas, são resultantes de eventos ocorridos durante o processo de regressão marinha. Os estudos desenvolvidos por Dalrymple et al. (1994), Harris et al. (2003, 2005) evidenciaram que estas estruturas de relevo positivas e negativas permanecem preservadas e, principalmente, controlam a distribuição dos aportes sedimentares 


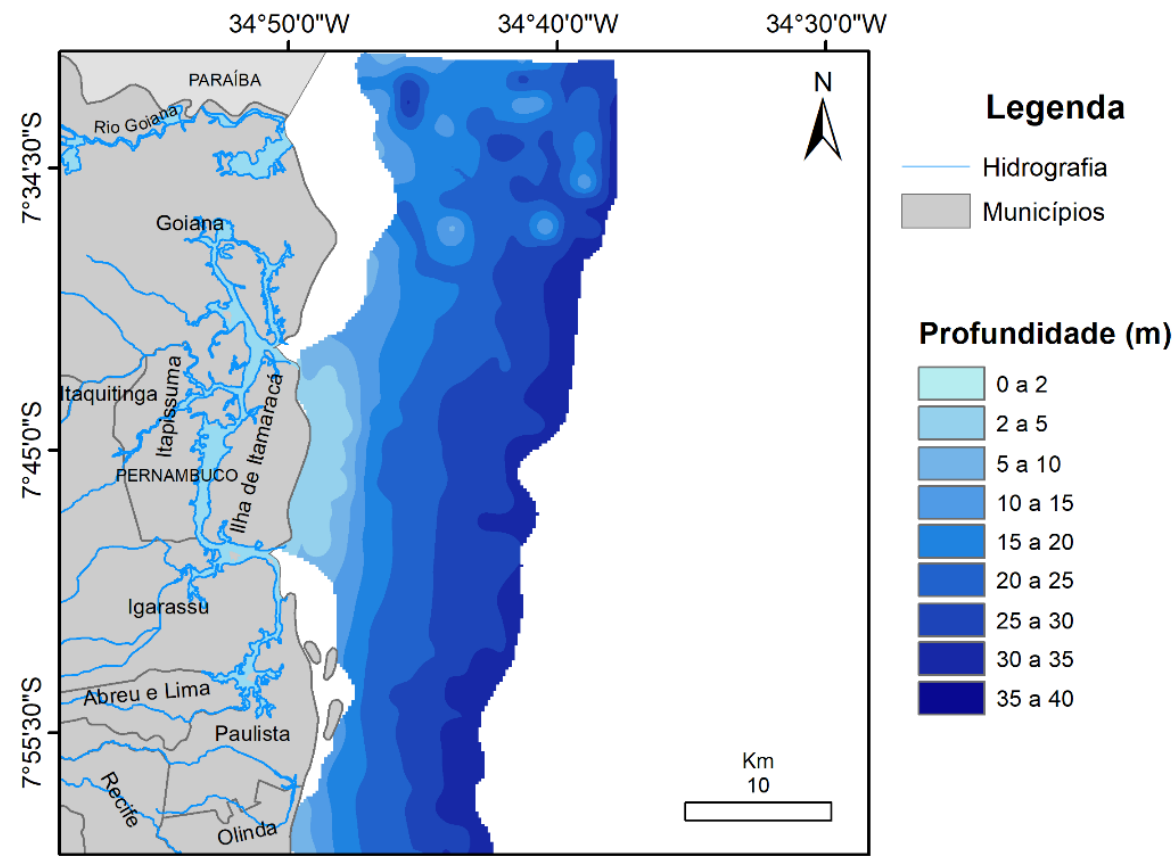

Figura 2 - Mapa batimétrico da área de estudo.

Ao comparar-se o resultado obtido com o estudo realizado por Camargo et al. (2015), observou-se que os canais encontrados estão associados às reentrâncias dos rios, como ocorre na região adjacente ao rio Sirinhaém, porção Sul da plataforma continental de Pernambuco. De acordo com Summerhayes et al., (1976), França (1979), Weschenfelder et al. (2008) e Conti \& Furtado (2009), estas depressões (paleocanais) são resultantes da erosão fluvial e subaérea que ocorreu durante o evento regressivo. Com base nestes autores, estas feições estão preservadas devido à cobertura sedimentar, semelhante às feições observadas por Harris et al. (2014) nas regiões de sedimentação carbonática da plataforma continental da Austrália, o que caracteriza, na área de estudo, o desenvolvimento de shelf valleys.Camargo et al. (2015) afirmaram que estas áreas possuem uma grande importância ecológica, pois propiciam uma série de habitats para organismos bentônicos de diversos níveis tróficos, de acordo com o tipo de substrato. Segundo estes autores, estas regiões além de seu destaque ecológico, em muitos casos são locais propícios para espécies de interesse pesqueiro e econômico.

\section{Caracterização Sedimentólógica}

\section{Diâmetro médio}

Com base na classificação do diâmetro médio de Folk \& Ward (1957) (Fig. 3) os depósitos superficiais apresentam um predomínio de grãos de classe areia muito grossa. Estes sedimentos apresentam uma combinação de areias quartzosas e de material de composição biogênica (Almeida, 2011; Oliveira, 2016). De acordo com Almeida (2018) a presença dos arenitos de praia e dos bancos de areia/cristas arenosas na plataforma interna dissipa a ação das ondas, deste modo possibilita a concentração desses materiais nas regiões centrais e norte da presente área de estudo.

No caso da deposição dos sedimentos finos nas regiões de depressões observadas próximo à linha de costa, sugere-se que este processo ocorre devido ao baixo aporte sedimentar na região de estudo, em decorrência do clima, o que gera um baixo aporte fluvial (Martins \& Coutinho, 1981; Vital et al. 2005; Conti, 2009; Conti \& Furtado, 2006; Blum et al., 2013; Harris et al., 2014; Camargo et al., 2015), e ao fato de que essas regiões mais profundas atuam como armadilhas para esses materiais 
devido a baixa hidrodinâmica de fundo (Kempf, 1970; Cramp et al. 1987; Granata et al. 1999; Liu et al., 2002; Vital et al, 2005).

\section{Grau de seleção}

De acordo com a classificação de Folk \& Ward (1957) (Fig. 4), observouse um gradiente negativo da seleção dos sedimentos. Os sedimentos muito bem selecionados estão localizados na parte norte da área de estudo, adjacentes à desembocadura do Rio Goiana. A diminuição do grau de seleção ocorre no sentido sudoeste, onde os sedimentos muito pobremente selecionados recobrem a região sul e a parte mais distante da linha de costa.

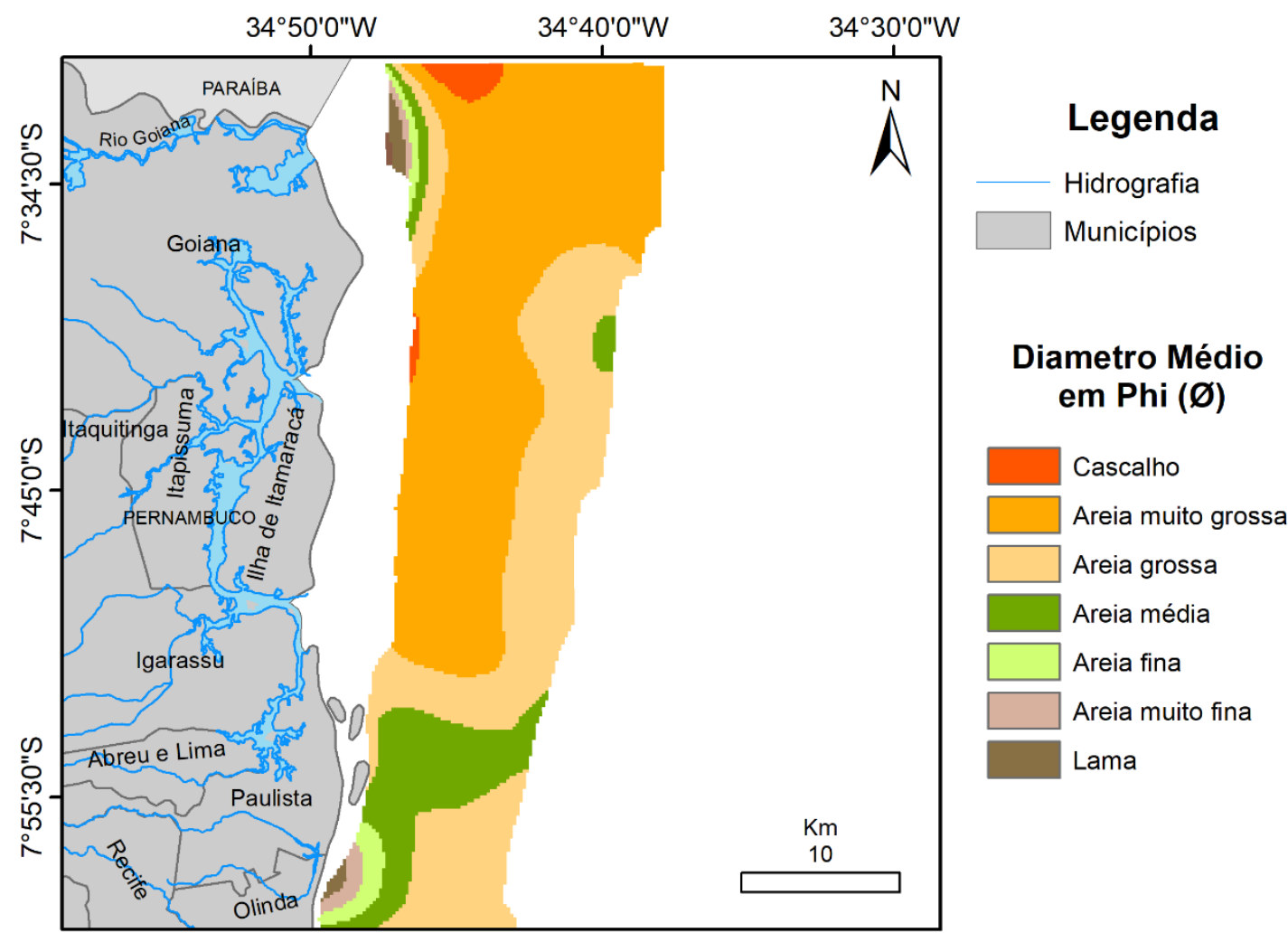

Figura 3 - Classificação do diâmetro médio das partículas sedimentares que compõem os sedimentos de fundo marinho na região de estudo (conforme Folk \& Ward, 1957). 


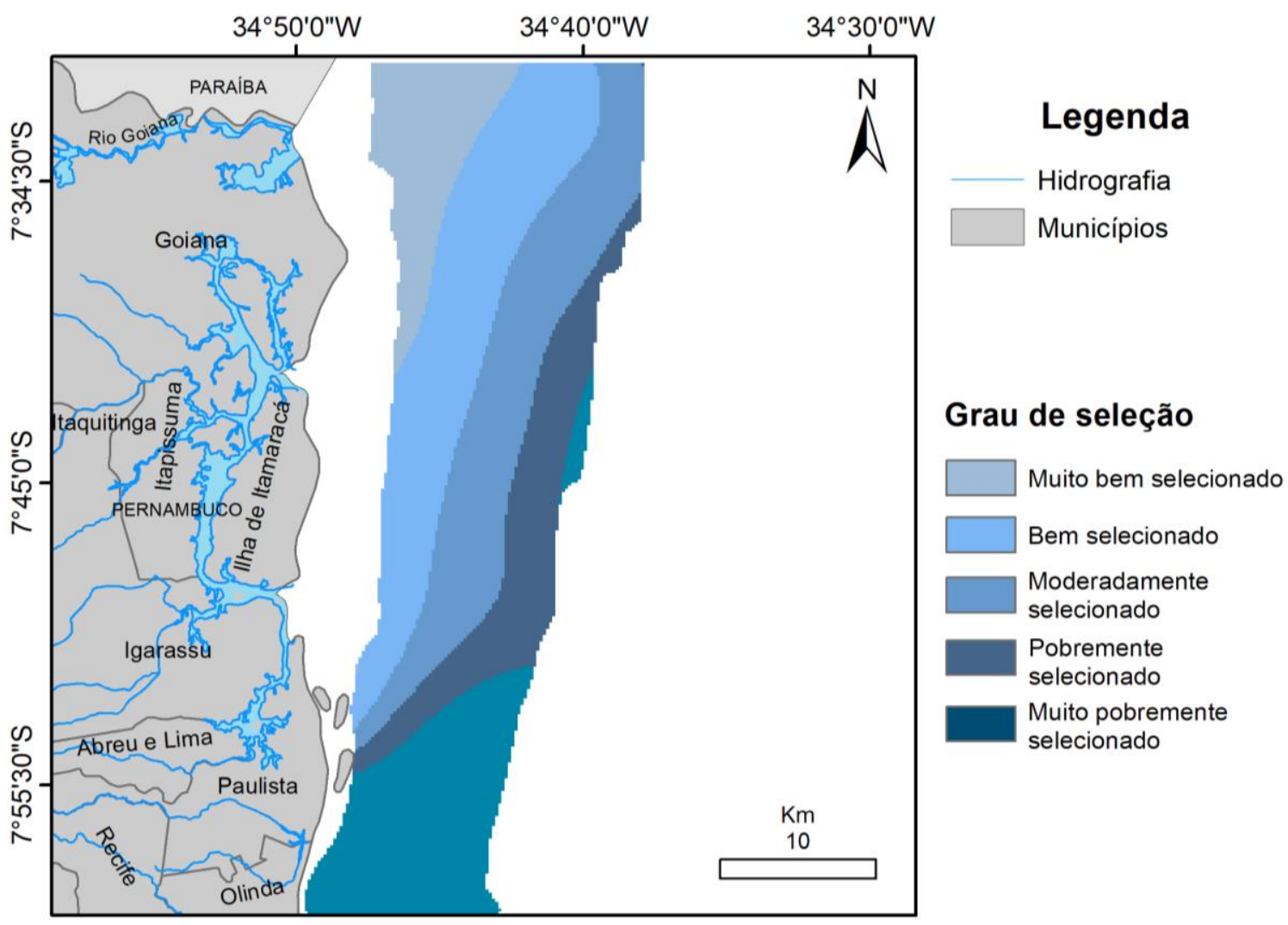

Figura 4 - Grau de seleção do sedimento que compõe as coberturas superficiais do fundo marinho na plataforma adjacente a região de Itamaracá (conforme Folk \& Ward, 1957)

Sahu (1964) afirma que o grau de seleção está associado à energia presente no meio. Com base no mapa batimétrico (Fig. 2) e os mapas batimétricos elaborados por Varela (2010), além da modelagem batimétrica apresentada por Almeida (2018), inferese que a presença dos arenitos em combinação com os paleovales são responsáveis pelo controle da energia hidrodinâmica do meio.

\section{Teor de Carbonato de cálcio}

A partir da classificação dos teores de carbonato (Fig. 5), com base em Larssoneur et al. (1982), foi possível definir que a região que apresenta menor cobertura de material carbonático (litobioclástico) é a zona costeira adjacente à Olinda. Também ocorre uma região com composição intermediária (biolitoclástico) localizada na região adjacente ao litoral de Paulista. Sugere-se que a maior quantidade de componentes terrígenos alóctones está atualmente relacionada ao aporte de origem fluvial (Oliveira et al., 2014; Barcellos et al., 2016; Santos \& Barcellos, 2017). Além disso, o aporte terrígeno está relacionado à erosão contínua de zonas de praia adjacentes (Manso et al., 2006). Estas regiões sofrem processos erosivos constantes, que acabam por fornecer material para a plataforma adjacente.

Sedimentos bioclásticos predominam em todo o restante da região da plataforma continental norte de Pernambuco. De acordo com a análise da composição dos sedimentos realizados por Barros et al. (2007), verificou-se que o material é composto por uma elevada carga de artículos de algas (Halimeda), conchas inteiras e fragmentos de gastrópodes, micro-gastrópodes, bivalves, microbivalves, foraminíferos e outros. Desta forma, é possível concluir que o sedimento que prevalece é de origem autóctone, cuja fonte é a produtividade primária local, e que a dinâmica local atua no retrabalhamento deste material. 


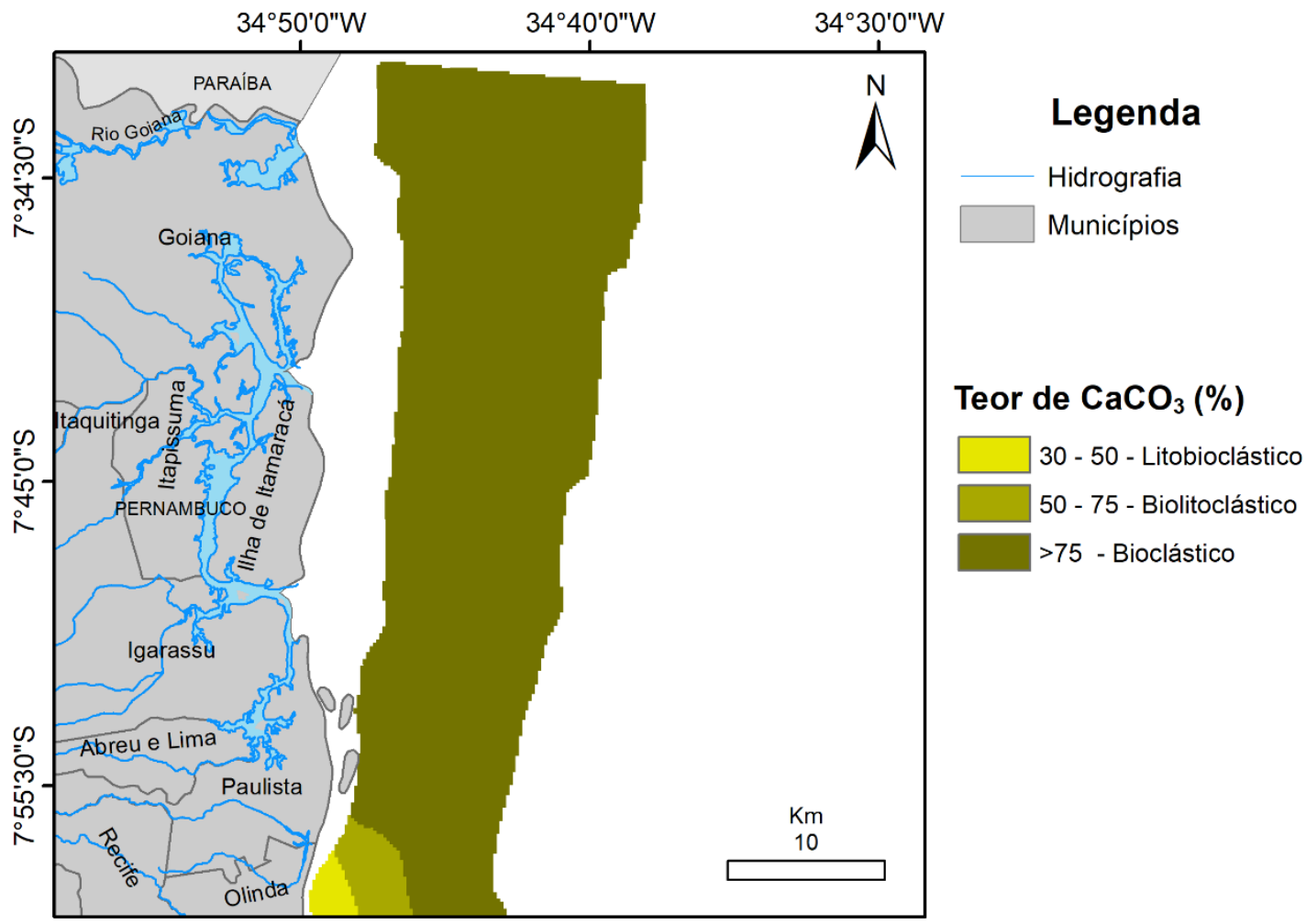

Figura 5 - Variação dos teores de $\mathrm{CaCO}_{3}(\mathrm{em} \%)$ de acordo com classificação de Larssoneur et al. (1982).

\section{Integração dos resultados}

Camargo et al. (2015) afirmam que o clima da região e das áreas onde as bacias costeiras estão inseridas não favorece um aporte considerável de material sedimentar terrígeno, deste modo há pouca contribuição, mesmo para a plataforma interna. Os sedimentos alóctones modernos são impedidos de atingir porções mais externas da plataforma por conta das diversas linhas de beachrocks dispostas paralelas à costa e presentes em diferentes profundidades $(2-5,16,22,25 \mathrm{~m})$ desde as porções mais rasas, conforme observado por Camargo et al. (2007) e Costa (2010). Isto fica evidenciado na figura 3 onde são observados 2 núcleos de acumulação de lama ao norte e ao sul adjacentes, respectivamente, ao sistema estuarino do Rio Goiana e ao complexo estuarino do Rio Capibaribe, associadas a paleovales que cortam estes alinhamentos de beachrocks. De fato, Pardal et al. (2019 in press) observou a deposição sazonal de lamas ao largo da desembocadura do sistema Capibaribe, incluindo estações de coleta mais ao norte adjacentes à Olinda, sugere que estas lamas são exportadas para o norte a partir da desembocadura do sistema, conforme o sentido das correntes predominantes na costa (SW-NE) (Domingues et al., 2017), mas também para leste condicionada pelo Canal de Olinda (paleovale do Rio Capibaribe) (DHN, 2019).

O baixo gradiente topográfico e aporte sedimentar terrígeno, a fisiografia acidentada e a alta produção biogênica algálica, o que se deve à presença mais marcada dos alinhamentos de beachrocks na plataforma centro-norte de Pernambuco, condiciona esta sedimentação predominantemente bioclástica areno-cascalhosa na quase totalidade da área de estudo (vide fig. 5). Estudos realizados por Santos e Barcellos (2017) e Oliveira et al (2017) indicam inclusive que alguns sistemas estuarinos pernambucanos, além de reter 
os terrígenos nas planícies de maré e manguezais, são também importadores de material biogênico da plataforma interna, como nos estuários do rio Formoso (litoral sul) e de Catuama/Itapessoca, respectivamente. Oliveira et al. (2017) observaram que durante a preamar sedimentos carbonáticos adentram no sistema estuarino de Catuama/Itapessoca. Ao comparar-se tais dados com os do presente trabalho, observa-se que os aportes terrígenos acabam aprisionados, sejam pelos ambientes estuarinos ou pelas feições geológicas - arenitos e paleocanais. Kempf (1970) observou que lamas terrígenas também ficariam aprisionadas entre as linhas de beach rocks, imprimindo esta característica biogênica à plataforma de Pernambuco, sendo esta considerada por fim como uma "plataforma faminta" (Dominguez et al.,1990).

\section{CONCLUSÕES}

Os resultados do estudo de aspectos batimétricos e da natureza da cobertura de sedimentos recentes na região plataformal rasa adjacente a Ilha de Itamaracá, mostraram que nesta área os sedimentos são predominantemente areno-cascalhosos carbonáticos. A distribuição dos sedimentos terrígenos e carbonáticos está associada à fisiografia da região, em conjunto com a hidrodinâmica e a produção biogênica local. Sugere-se que os arenitos praiais (beachrocks) que ocorrem na linha de litoral controlam o efeito de energia das ondas e marés o que interfere no transporte de sedimentos terrígenos. Também, é sugerido que as regiões de paleocanais (Shelfvalleys) servem como uma zona de armadilha para os sedimentos mais finos, principalmente junto à costa, conforme observado ao largo dos estuários dos rios Goiana e Capibaribe.
Sugere-se também que devido ao aporte reduzido de terrígenos para a plataforma ocorreu a preservação de feições como os alinhamentos de beachrock e paleovales submersos. Para uma melhor compreensão acerca das estruturas morfológicas e sedimentos observados, faz-se necessário estudos mais detalhados advindos de dados de sonografia de varredura lateral sonar multi-feixe, de sísmica rasa, de novas coletas diretas de sedimentos de superfície de fundo, bem como de estimativas de carga sedimentar de aporte de sedimentos para a plataforma.

\section{Agradecimentos}

A autora agradece à CAPES pela concessão da bolsa de doutorado. Os autores agradecem Serviço Geológico do Brasil - CPRM que por meio do projeto Granulados Marinhos - GRANMAR Pernambuco pela coleta e armazenamento das amostras, à Hortência M. B. de Assis e equipe (CPRM-PE), Ronaldo G. Bezerra (CPRM-PE) pela concessão dos dados coletados in loco, a Carlos Fernando de A. Soares Júnior (UFC) e Prof. George Satander Sá Freire (UFC) pela concessão das análises sedimentares realizadas. Ao Laboratório de Geologia e Geofísica Marinha e ao Prof. Valdir Manso pelos dados extras de batimetria.

\section{REFERÊNCIAS}

Almeida, T. L. M. 2011. Sedimentologia e batimetria da plataforma interna adjacente a ilha de Itamaracá-PE. Dissertação de Mestrado. PPGEOUFPE. 92p.

Almeida, T. L. D. M. 2018. Caracterização da dinâmica costeira de Itamaracá-PE: aplicação do sistema de modelagem costeira do Brasil. Tese de doutorado. PPGEOUFPE. 183p. 
Araújo, T.C.M., Seoane, J.C.S., Coutinho, P.N. 2004. Geomorfologia da plataforma continental de Pernambuco. In: Leça, E.E., Neumann-Leitão, S., Costa, M.F. (Eds.), Oceanografia-um Cenário Tropical. Ed. Bagaço, Recife, pp. 39-57.

Barcellos, R. L., Alves, C. S., \& Fetter Filho, A. F. H. 2016. Geoquímica e Dinâmica Sedimentar do Sistema Estuarino do Rio Goiana. 1. ed. Saarbrücken, Alemanha: NEAEdições, 2016b. v. 1. 89p.

Barros, L. C., Valença, L. M. M., Manso, V. D. A. V., Madruga Filho, J. D., \& Oliveira, J. A. R. 2007. Textura, composição e arredondamento dos sedimentos da plataforma continental interna adjacente às desembocaduras sul do canal de Santa Cruz e do Rio Timbó, norte do Estado de Pernambuco. Estudos Geológicos, 17(1), 58-70.

Blum, M.D., Martin, J., Miliken, K., Garvin, M. 2013. Palleovaley systems: Insights from Quaternary analogs and experiments. Earth Sci. Rev. 116, 128-169.

Camargo, J.M.R., Araújo, T.C.M., Maida, M., Ushizima, T.M., 2007. Morfologia da plataforma continental interna adjacente $\mathrm{o}$ município de Tamandaré, sul de Pernambuco-Brasil. Rev. Bras. Geofís. 25 (Supl. 1), 79-89.

Camargo, J. M. R.; Araújo, T. C. M.; Ferreira, B. P.; Maida, M. 2015. Topographic features related to recent sea-level history in a sediment-starved tropical shelf: Linking the past, present and future. Regional Studies in Marine Science 2: 203-211.

Carver, R. E. 1971. Procedures in Sedimentary Petrology. Edited by Robert E. Carver, University of Georgia-EUA. 652p.
Clark, P.U., Dyke, A.S., Shakun, J.D., Carlson, A.E., Clark, J., Wohlfarth, B., Mitrovica, J.X., Hostetler, S.W., McCabe, A.M. 2009. The last glacial maximum. Science 325, 710-714

Conti, L. A. 2009. Evidências da evolução dos sistemas de paleodrenagens na plataforma continental da região de São Sebastião (litoral norte do estado de São Paulo). Revista Brasileira de Geomorfologia, 10(2) 44-55.

Conti, L. A., \& Furtado, V. V. 2006. Geomorfologia da plataforma continental do Estado de São Paulo. Revista Brasileira de Geociências, 36(2), 305-312.

Conti, L. A., \& Furtado, V. V. 2009. Topographic registers of paleovalleys on the southeastern Brazilian continental shelf. Brazilian Journal of Oceanography, 57(2), 113-121.

Costa, M. B. S. F. (2010). Influência da estrutura recifal na transformação das ondas do litoral de Recife e Jaboatão dos Guararapes/PEBrasil. Tese de mestrado do Programa de Pós-Graduação em Oceanografia, Universidade Federal de Pernambuco. 92f.

Coutinho, P. N. 1996. Levantamento do estado da arte da pesquisa dos recursos vivos marinhos do BrasilOceanografia Geológica. Região Nordeste. Programa REVIZEE. Ministério do Meio Ambiente, dos Recursos Hídricos e da Amazônia Legal (MMA). Brasília, 97pp.

CPRH, 2001. Diagnóstico sócioambiental do Litoral Sul de Pernambuco. Companhia Pernambucana do Meio Ambiente. Disponível em: < http://www.cprh.pe.gov.br/downl oads/2diagnostico_ambiental.pdf> . Acesso em: 04 julho de 2019.

CPRH. 2003. Diagnóstico Socioambiental do litoral norte do 
litoral norte de Pernambuco. Companhia Pernambucana do Meio Ambiente. Recife. (2003). $214 \mathrm{p}$

CPRM. 2018. SERVIÇO GEOLÓGICO DO BRASIL. Relatório Técnico do Projeto Granulados Marinhos do Brasil - GRANMAR. Disponível em: <http://www.cprm.gov.br/geo/pgb /inicio/Geol\%20Geof\%20Marinha .pdf>. Acesso em 19 jul. 2019.

Cramp, A., Collins, M.B., Wakefield, S.J. 1987. Sedimentation in the Zakynthos channel-a conduit link to the Hellenic Trench, eastern Mediterranean. Mar. Geol. 76, 7187.

DHN. 2019. Porto do Recife - Carta náutica 902. Departamento de Hidrografia e Navegação. Marinha do Brasil. Disponível em: $<$ https://www.marinha.mil.br/chm /dados-do-segnav-cartasraster/porto-do-recife>

Domingues, E. D. C., Schettini, C. A. F., Truccolo, E. C., \& Oliveira Filho, J. C. D. 2017. Hydrography and currents on the Pernambuco Continental Shelf. RBRH, 22. e43.

Dominguez, J. M. 2009. The coastal zone of Brazil. In Geology and geomorphology of holocene coastal barriers of Brazil (pp. 1751). Springer, Berlin, Heidelberg.

Dominguez, J. M., L., Bittencourt, A. C. S. P., Leão, Z. M. A. N. 1990. Geologia do Quaternário costeiro do estado de Pernambuco. Revista Brasileira de Geociências 20: 208215

Flores-Montes, M. J.; Macêdo, S. J.; Koening, M. L.; Lins Correa, I. 1998. Variação nictemeral do fitoplancton e elementos nutrientes no Canal de Santa Cruz, Itamaracá - PE - Brasil. Trab. Oceanogr. Univ. Federal.PE, Recife,v.26, n. 1, p. 13-26.
Folk, R. L., \& Ward, W. C. 1957. Brazos River bar: a study in the significance of grain size parameters. Journal of Sedimentary Research, 27(1).

França, A.M.C., 1979. Geomorfologia da margem continental leste brasileira e da bacia oceânica adjacente. In: CENPES/DINTEP, Geomorfologia da Margem Continental Brasileira e das áreas Oceânicas Adjacentes PETROBRAS, Rio de Janeiro, pp. 89-127.

Gardner, J. V., Calder, B. R., Hughes Clarke, J. E., Mayer, L. A., Elston, G., Rzhanov, Y., 2007. Drowned shelf-edge deltas, barrier islands and related features along the outer continental shelf north of the head of De Soto Canyon, NE Gulf of Mexico. Geomorphology 89, 370390.

Gaspar, F. L. 2009. Análise da concentração de fósforo em sedimentos dos rios Botafogo e Carrapicho, no Sistema estuarino do Canal de Santa Cruz, Itamaracá, PE. Dissertação de mestrado. UFPE

Granata, T.C., Vidondo, B., Duarte, C.M., Satta, M.P., Gracia, M. 1999. Hydrodynamicsand particles transport associated with a submarine canyon of Blanes (Spain), NW Mediterranean Sea. Cont. Shelf Res. 19, 1249-1263.

Hayes, M. O., 1979. Barriers islands morphology as a function of tidal and wave regime. New York: (Ed.) Barrier Island from the Gulf of Mexico.

Heezen, B. C., Glass, B., \& Menard, H. W. 1966. The Manihiki Plateau. In Deep Sea Research and Oceanographic Abstracts (Vol. 13, No. 3, pp. 445-458). Elsevier.

Harris, P.T., Macmillan-Lawler, M., Rupp, J., Baker, E.K. 2014. 
Geomorphology of the oceans. Mar. Geol. 352, 4-24.

Kempf, M. 1970. Nota Preliminar Sobre Fundos Costeiros da Região de Itamaracá (Norte do Estado de Pernambuco, Brasil). Tropical Oceanography-ISSN: 1679-3013, 9(1).

Larssoneur, C.; Bouysse, P. \& Aufret, J.P. 1982. The surpeficial sediments of the English Channel and its western approaches.Sedimentology, 29(6):851-864.

Liu, J.T., Liu, K., Huang, J.C. 2002. The effect of a submarine canyon on the river sediment dispersal and inner shelf sediment movements in southern Taiwan. Mar. Geol. 181, 357-386

Manso, V.A.V., Kima Filho,M.F., Oliveira,A.T. 1992.

Macrozoneamento costeiro do Litoral de Pernambuco - Folha Itamaracá - SB.25-Y-C-VII. Relatório técnico. Recife. Laboratório de Geologia e Geofísica Marinha - LGGM. Departamento de Engenharia de Minas - DEMINAS UFPE/CPRH.

Manso, V. D. A. V., Coutinho, P. N., Guerra, N. C., \& Junior, C. F. A. S. 2006. Erosão e Progradação do Litoral Brasileiro: Pernambuco. Laboratório de Geologia e Geofísica Marinha-LGGM. Recife: Editorial da Universidade, 162.

Martins, L. R., \& Coutinho, P. N. 1981. The Brazilian continental margin. Earth-Science Reviews, 17(1-2), 87-107.

Medeiros, C., \& Kjerfve, B. 1993. Hydrology of a tropical estuarine system: Itamaracá, Brazil. Estuarine, Coastal and Shelf Science, 36(5), 495-515.

Oliveira, L. E. E. 2016. Estudo da matéria orgânica sedimentar como indicador ambiental nas adjacências do Porto do Recife, Ilha de Itamaracá e arquipélago de Fernando de Noronha (Pernambuco).

Oliveira, L. E. E., dos Santos, L. D., Montes, M. D. J. F., \& Barcellos, R. L. 2017. INFLUÊNCIA DA MARÉ NA VARIABILIDADE SEDIMENTAR DA BARRA DE CATUAMA, ILHA DE ITAMARACÁ, PERNAMBUCOBRASIL. Estudos Geológicos, 27, 2.

Oliveira, T. D. S., Barcellos, R. L., Schettini, C. A. F., \& Camargo, P. B. D. 2014. Processo sedimentar atual e distribuição da matéria orgânica em um complexo estuarino tropical, Recife, PE, Brasil. Revista de Gestão Costeira Integrada, 14(3), 399-412.

Oliveira Filho, J. C. 2015. Circulação e transporte de sal e sedimentos me suspensão no Sistema Estuarino da Ilha de Itapessoca (PE), Brasil.Dissertação de mestrado DOCEAN, UFPE, Recife, 59p.

Pardal, E. C., Xavier, D. A., Vilela, I. M., Camargo, P. B., Flores-Montes, M. J., Barcellos, R. L. (2019). Variabilidade sedimentológica e geoquímica em sistema estuarino tropical sob forte influência antrópica no nordeste brasileiro (rio Capibaribe-PE). In press.

Ponzi, V. R. A. 2004. Sedimentação marinha. BAPTISTA NETO, JA, PONZI, VR A., SICHEL, SE Introdução à Geologia Marinha. Rio de Janeiro: Interciência, 219241.

Ratisbona, L. R. (1976). The climate of Brazil. Climates of Central and South America, 12, 219-293.

Sahu, B. K. 1964. Depositional mechanisms from the size analysis of clastic sediments.Journal of Sedimentary Research, 34(1). 
Salzmann, L., Green, A., Cooper, J.A., 2013. Submerged barrier shoreline sequences on a high energy, steep and narrow shelf. Mar. Geol. 346, 366-374.

Santos, L. D., \& Barcellos, R. L. 2017. Sedimentação Atual do Estuário do Rio Formoso-PE (Brasil). Saarbrucken, Germany: NEAEdições, 113p.

Silva, L.A. 2004. Sedimentologia do Canal de Santa Cruz - Ilha de Itamaracá - PE. Dissertação de Mestrado. PPGEO-UFPE.

Silva, F.A.N. 2012. Análise do comportamento sedimentológico e hidrdinâmico da desembocadura norte do canal de Santa Cruz - PE. Dissertação de mestrado em Geologia. PPGEO-UFPE.

SUDENE, $1978 . \quad$ Inventário Hidrogeológico básico do Nordeste: Folha ${ }^{\circ} 21$, Recife- NO. Recife, 183p.

Suguio, K.1973. Introdução à sedimentologia. São Paulo: Edgard Blücher,. $317 \mathrm{p}$.

Suguio, K. 2003. Geologia sedimentar. Edgard Blücher. 400p.

Summerhayes, C.P., Fainstein, R., Ellis, J.P., 1976. Continental margin off Sergipe and Alagoas, Northeastern Brazil: a reconnaissance geophysical study of morphology and structure. Mar. Geol. 20, 345361.

Varela, G. J. A. C.. 2010. Influência dos recifes na evolução da linha de costa na Ilha de Itamaracá- PE. Dissertação (Mestrado)
Universidade Federal de Pernambuco. CTG. Programa de Pós- -Graduação em Geociências, Recife, p. 125. 2010.

Vital, H., Silveira, I. M. D., \& Amaro, V. E. 2005. Carta sedimentólogica da plataforma continental brasileira: área Guamaré a Macau (NE Brasil), utilizando integração de dados geológicos e sensoriamento remoto. Revista Brasileira de Geofísica, 23(3), 233-241.

Wagle, B.G., Veerayya, M., 1996. Submerged sand ridges on the western continental shelf off Bombay, India: evidence for Late Pleistocene-Holocene sea-level changes. Mar. Geol. 136, 79-95.

Weschenfelder, J., Corrêa, I.S.C., Toldo Junior, E.E., Baitelli, R. 2008. Paleocanais como indicativo de eventos regressivos quaternários do nível do mar no sul do Brasil. Rev. Bras. Geofís. 26 (3), 367375.

Yokoyama, Y., Lambeck, K., De Deckker, P., Johnston, P., \& Fifield, L. K. 2000. Erratum: Timing of the Last Glacial Maximum from observed sea-level minima. Nature, 406(6797), 713716.

Yokoyama, Y., Esat, T. M., Thompson, W. G., Thomas, A. L., Webster, J. M., Miyairi, Y., ... \& Fallon, S. (2018). Rapid glaciation and a two-step sea level plunge into the Last Glacial Maximum. Nature, 559(7715), 603. 